University of Nebraska - Lincoln

DigitalCommons@University of Nebraska - Lincoln

\title{
Meiotic Recombination, Cross-Reactivity, and Persistence in Plasmodium falciparum
}

\author{
F. Ellis McKenzie \\ Harvard University \\ Marcelo U. Ferreira \\ Faculdade de Medicina de Sao Jose do Rio Preto \\ J. Kevin Baird \\ U.S. Naval Medical Research Unit \# 2, jkevinbaird@yahoo.com \\ Georges Snounou \\ Institut Pasteur \\ William H. Bossert \\ Harvard University
}

Follow this and additional works at: https://digitalcommons.unl.edu/usnavyresearch

McKenzie, F. Ellis; Ferreira, Marcelo U.; Baird, J. Kevin; Snounou, Georges; and Bossert, William H., "Meiotic Recombination, Cross-Reactivity, and Persistence in Plasmodium falciparum" (2001). U.S. Navy Research. 68.

https://digitalcommons.unl.edu/usnavyresearch/68

This Article is brought to you for free and open access by the U.S. Department of Defense at DigitalCommons@University of Nebraska - Lincoln. It has been accepted for inclusion in U.S. Navy Research by an authorized administrator of DigitalCommons@University of Nebraska - Lincoln. 


\title{
MEIOTIC RECOMBINATION, CROSS-REACTIVITY, AND PERSISTENCE IN PLASMODIUM FALCIPARUM
}

\author{
F. Ellis McKenzie, ${ }^{1}$ Marcelo U. Ferreira, ${ }^{2}$ J. Kevin Baird ${ }^{3}$ Georges Snounou, ${ }^{4}$ And \\ William H. BosserT ${ }^{1}$ \\ ${ }^{1}$ Department of Organismic and Evolutionary Biology and Division of Engineering and Applied Sciences, Harvard University, \\ 142 Maxwell-Dworkin Laboratory, 33 Oxford Street, Cambridge, Massachusetts 02138 \\ ${ }^{2}$ Laboratorio de Parasitologia Molecular, Faculdade de Medicina de Sao Jose do Rio Preto, Sao Jose do Rio Preto (SP), Brazil \\ ${ }^{3}$ Parasitic Diseases Program, United States Naval Medical Research Unit 2, Jakarta, Indonesia \\ ${ }^{4}$ Unite de Parasitolgie Biomedicale, Institut Pasteur, Paris, France
}

\begin{abstract}
We incorporate a representation of Plasmodium falciparum recombination within a discrete-event model of malaria transmission. We simulate the introduction of a new parasite genotype into a human population in which another genotype has reached equilibrium prevalence and compare the emergence and persistence of the novel recombinant forms under differing cross-reactivity relationships between the genotypes. Cross-reactivity between the parental (initial and introduced) genotypes reduces the frequency of appearance of recombinants within three years of introduction from $100 \%$ to $14 \%$, and delays their appearance by more than a year, on average. Cross-reactivity between parental and recombinant genotypes reduces the frequency of appearance to $36 \%$ and increases the probability of recombinant extinction following appearance from $0 \%$ to $83 \%$. When a recombinant is cross-reactive with its parental types, its probability of extinction is influenced by cross-reactivity between the parental types in the opposite manner; that is, its probability of extinction after appearance decreases. Frequencies of $P$. falciparum outcrossing are mediated by frequencies of mixed-genotype infections in the host population, which are in turn mediated by the structure of cross-reactivity between parasite genotypes. The three leading hypotheses about how meiosis relates to oocyst production lead to quantitative, but no qualitative, differences in these results.
\end{abstract}

Key words._-Infectious disease, malaria, parasite, Plasmodium falciparum, transmission.

Received December 12, 2000. Accepted March 29, 2001.

The life cycle of Plasmodium falciparum, the major cause of malaria morbidity and mortality worldwide, involves obligate sexual recombination, which occurs within bloodmeals taken by Anopheles mosquitoes from infectious humans. The frequency with which these $P$. falciparum matings involve outcrossing rather than selfing, and thus the degree to which the species is characterized by panmixia rather than facultative clonality, has been vigorously debated for a decade, almost exclusively at the two extremes (Ayala et al. 1998; Walliker et al. 1998). However, recent evidence suggests that inferred $P$. falciparum population structures may differ between sites and times and seem to exhibit intermediate levels of outcrossing (Paul et al. 1999; Anderson et al. 2000a). Not surprisingly, these conflicting analyses are complicated by their dependence on the specific loci examined, and in particular by the degree to which these markers may engage host immunological responses.

Plasmodium infection of a vertebrate begins with a small inoculum of sporozoites from the salivary glands of a bloodfeeding Anopheles mosquito. Sporozoites penetrate liver cells, transform, and multiply to produce thousands of free merozoites. Each of these asexual merozoites invades a red blood cell, completes another phase of multiplication, then bursts the cell, releasing eight to 32 more merozoites to invade more red blood cells. This blood cycle may be repeated many times, in the course of which some of the invading merozoites instead develop into the sexual, nonreplicating transmission stages known as gametocytes. If gametocytes are taken up by a feeding Anopheles, gametes from male and female gametocytes may unite to form zygotes, and, in turn, the oocysts from which infective sporozoites arise. The zygote is the sole diploid stage in the Plasmodium life cycle:
Meiosis occurs within a few hours of zygote formation, and the remaining weeks or months of the life cycle consist of haploid forms.

Thus, the basic biology of Plasmodium argues that frequencies of parasite transmission between host and vector constitute one set of bounds for recombination rates, and that numbers and relative densities of infectious genotypes within a host constitute one set of bounds for outcrossing events. Although mechanisms for even these minimal constraints are largely unknown, it seems certain that immunological responses are strongly influential, such that some parasite genotypes might not co-occur in humans or transit to mosquitoes independently (Ferreira et al. 1998; Gilbert et al. 1998; Healer et al. 1999; Snounou et al. 1999). It is particularly important to understand the dynamics of interactions between human immunity and parasite recombination because, by direct selection or by altering human immune responses, infectivity, or other factors, antimalarial vaccines or drugs may alter patterns of parasite recombination so as to either hasten or retard the decline of their own efficacies (Buckling et al. 1999; Baird 2000).

Discrete-event models of malaria transmission adapt the population-compartment scheme of classic models to the level of individuals within interacting host and vector populations (Fig. 1). They embed simple, explicit models of $P$. falciparum life-cycle dynamics within individual humans and individual mosquitoes in thousands of interacting representations of individual humans and individual mosquitoes (McKenzie et al. 1998, 1999). This approach intrinsically encompasses multiple interactions among $P$. falciparum variants, their partitioning across individual human characteristics and infection histories, and their sampling through mosquito transmission. 

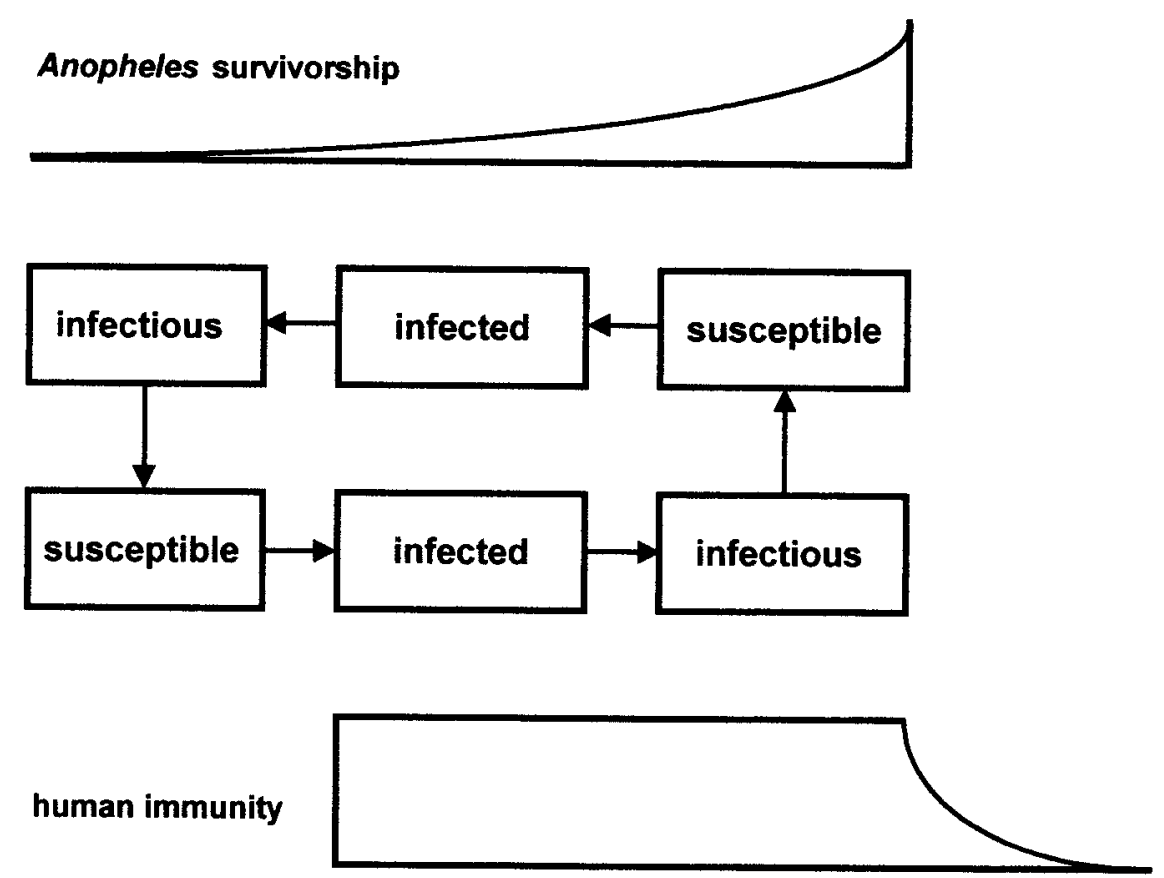

FIG. 1. A schematic of the discrete-event model, representing the status of a female Anopheles mosquito (top) and of a human (bottom) with respect to appropriate stages of the Plasmodium falciparum life cycle. Vector bloodmeals allow parasite transmission, linking the two phases. McKenzie et al. (1998) provides a detailed description.

The major conclusion from our basic model was that the daily mortality of the vector and the duration of human infectivity influence malaria prevalence in a human population more strongly than does the duration of human immunity to reinfection. Our results agreed with those of the classic differential-equation models of malaria, where they were comparable, but we also demonstrated that ostensibly identical representations of human immunity in differential-equation, differential-delay-equation, and discrete-event models may in fact be incommensurable. An expanded model encompassed the transmission of multiple parasite phenotypesphenotypes either fully independent or interactive through complete cross-reactivity. With independent phenotypes, mixed infections occurred in humans at the frequencies expected under standard statistical hypotheses of independence, whereas with cross-reactivity, mixed infections occurred at frequencies far below those expected; in either case, mixed infections occurred in mosquitoes at frequencies above those expected (in line with field data on mixed-species Plasmodium infections; McKenzie and Bossert 1997a, 1999). This juxtaposition suggested intriguing implications for outcrossing rates, in that with independent phenotypes, a mixed infection in a human might derive from sequential infectious bites or from a single multiply infectious bite, whereas with cross-reactivity it could derive only from the latter. Accordingly, here we expand our modeling framework to investigate recombination among $P$. falciparum genotypes and how its characteristics might be influenced by specific structures of cross-reactivity.

\section{Materials AND Methods}

Our discrete-event models of $P$. falciparum transmission use a single timeline variable to track each parasite life cycle within and between individuals (Fig. 1). We have previously described the assumptions, implementation, and behaviors of the model in detail and compared them to those of classic malaria-transmission models (McKenzie et al. 1998, 1999).

Within the model, a given number of humans and mosquitoes are drawn at random each day, with replacement, for interaction through the taking of bloodmeals. Following Macdonald (1957) and our previous work, we fix the number of such bites per day at one-half the number of vectors and assume that humans are the sole source of bloodmeals. We generally assume a 10:1 ratio of mosquitoes to humans, again following Macdonald (but see McKenzie et al. 1998). The mosquito-dynamics component of the model assumes a constant daily probability of death (parameter range 0.0460.139 , half-life five to 15 days, in accord with field data), and in our previous work each dead mosquito was replaced with one newly emerged adult. Here each dead mosquito is replaced by a number of newly emerged adults that is a Poisson random variable with parameter $K / N$, where $N$ is the current mosquito population size and the value of $K$ a parameter in the model (set here at 5000). Because the number of mosquito deaths in a day is generally large $(>10)$, we can approximate the Poisson by a normal random variable with mean and variance $K / N$, rounding the result to an integer (Haight 1967). The effect of density dependence swamps the stochastic fluctuations, so that even in very small mosquito populations $(\sim 25)$ the probability of vector extinction is near zero.

The model defines immunity in terms of an individual human's susceptibility to reinfection with a given parasite entity, determined by a daily decay of protection after clearance of infection with that entity. The absence of definitive empirical data makes it difficult to determine a relevant param- 
mo

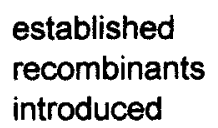

established recombinants introduced

\section{$\mathbf{a b}$}

m1

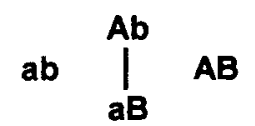

m2

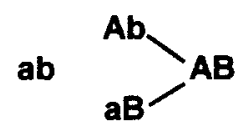

m3

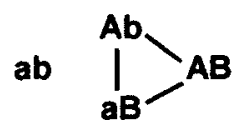

m4

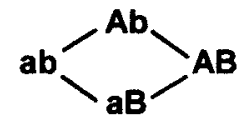

m5

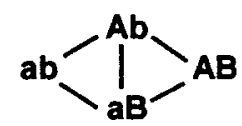

FIG. 2. The six cross-immunity matrices, m0-m5, with the four distinct genotypes in each given as a two-locus, two-allele system Ab, $\mathrm{aB}, \mathrm{AB}, \mathrm{ab}$. The two parental genotypes are the initial, equilbrium $\mathrm{Ab}$ and the introduced $\mathrm{aB} ;$ the two recombinants are $\mathrm{ab}$ and $\mathrm{AB}$. Note that matrices $\mathrm{m} 0, \mathrm{~m} 1, \mathrm{~m} 4$, and $\mathrm{m} 5$ are symmetric with respect to cross-immunity between genotypes, whereas matrices $\mathrm{m} 2$ and $\mathrm{m} 3$ are asymmetric.

eter range under this operational (or any other) definition of human immunity in malaria. Our previous work focussed on 100 days as a standard half-life of immunity and systematically explored the effects of values ranging from 50 to 150 days. Here we set 2000 days as a standard half-life. With this one change, we repeated all previous runs and systematically explored the effects of half-lives ranging from 1000 to 3000 days. Each prevalence-response surface proved to be lower than those with the shorter immunity half-lives, but remained well within the bounds of field data from perennialtransmission regions and fully in accord with the qualitative conclusions of our previous work.

The model defines cross-reactivity between parasite entities as complete and reciprocal, that is, entities that are crossreactive share every aspect of a host's immune-response dynamics except, in general, the phenotype-specific value of the immune-decay parameter. Thus, the probability that a human individual becomes infected when challenged by inoculation depends not only on those parasite entities actually in the inoculum or those with which the individual was most recently infected, but on all those with which each is connected through cross-reactivity. The probability of (re)infection with each entity is calculated in accord with the lowest probability of infection; that is, for each genotype in an inoculum, a probability of infection is calculated for all genotypes within its cross-reactivity group in the challenged individual's infection history. Then for each genotype in the inoculum, independently, the lowest of these probabilities is taken as the probability of infection for that genotype in a Bernoulli trial. We represent each possible combination of the presence and absence of cross-reactivity among the four genotypes as a pattern of ones and zeros in a $4 \times 4$ matrix, and refer to each distinct combination by a matrix number. The dynamic repertoire of the system could be increased by incorporating fractional or asymmetric values, but there is as yet no clear empirical basis for doing so with respect to malaria. Here we set the values of the immune-decay parameter for the four genotypes equal, then consider six crossreactivity matrices, $\mathrm{m} 0-\mathrm{m} 5$ (Fig. 2); we refer to the four distinct genotypes in each by the notation $\mathrm{Ab}, \mathrm{aB}, \mathrm{AB}, \mathrm{ab}$. Note that matrices $\mathrm{m} 0, \mathrm{~m} 1, \mathrm{~m} 4$, and $\mathrm{m} 5$ are symmetric with respect to cross-reactivity between genotypes, whereas matrices $\mathrm{m} 2$ and $\mathrm{m} 3$ are asymmetric.

We have modeled the life history of the parasite in the vector in more detail than in previous work. This resolves a discrepancy between our earlier models and field data-substituting a probability for the certainty that a mosquito feeding on a gametocyte carrier becomes infected-and allows us to represent the dynamics of recombination between $P$. falciparum genotypes, initially within a two-locus, two-allele-per-locus system. An additional parameter in the expanded model represents a recombination rate between the loci, $0 \leq r \leq 1$. For each mosquito bloodmeal taken, the probability distribution of oocyst genotypes that might result from the bloodmeal is calculated using the given value of $r$ and the distribution of gametocyte genotypes in the bloodmeal. The number of oocysts resulting from the bloodmeal is a Poisson random variable with parameter 0.8 (in accord with data on naturally infected Anopheles gambiae; Pringle 1966; Haji et al. 1996); a sample of that size is drawn from the probability distribution of genotypes calculated above. With this representation, on average roughly $55 \%$ of mosquito feeds on gametocyte carriers produce oocysts, again in line with field data (Carter and Graves 1988; Toure et al. 1998). Note that each genotype is considered as present in the given gametocyte pool or not; relative densities of the gametocyte genotypes do not enter into the calculation of the ookinete frequencies. When a mosquito is superinfected, any resulting oocysts are added to those already present from prior infections; thus, all else being equal, older mosquitoes will have higher oocyst densities.

With this additional change, with $r=0$, we repeated all previous runs, and found the prevalence surfaces lower but still clearly in accord with field data. Two qualitative changes from our previous conclusions emerged, each of which follows logically from more realistic mosquito-infection probabilities: (1) with fully independent phenotypes, the relative influence of vector mortality on overall prevalence is, on average, clearly greater than that of the duration of host infectivity; and (2) with fully cross-reactive phenotypes, the relative influence of the duration of host immunity is, on average, clearly equal to that of the duration of human infectivity. We also found that with parameter sets that combine the maximum vector mortality, the minimum duration of infectivity, and the maximum duration of immunity $(0.139,10$, 3000 ), spontaneous extinctions of the parasite occurred within three years in up to $20-25 \%$ of runs.

We set the host population size at 500, fix the intervals 
required to progress from an infected to an infectious state at 10 and 20 days for the vector and host, respectively, and allow the duration of host infectivity to range from 10 to 30 days (McKenzie et al. 1998, 1999). We initiate each replicate run with none of the vectors infected and with $15 \%$ of the hosts infected with a single genotype. Within two years, this initial genotype reaches equilibrium prevalence; we then introduce a second genotype, in the form of a single infectious human, and continue the run for three more years. Below we refer to the initial, equilibrium, and the introduced genotypes jointly as the "parental', genotypes, and to their distinct progeny jointly as the "recombinants."

For each of the replicate runs, for each cross-reactivity matrix, for each recombinant form we recorded the number of runs in which it appeared in at least one human infection, the day of its (first) appearance, and its average prevalence over the final year. Each prevalence figure below encompasses the infected and the infectious states and represents an average over all replicates for a given parameter set (standard deviations $<0.01$ ). All prevalence figures are calculated over the fifth and final year; they exclude runs in which parasite extinction occurred during the first four years, but include those in which extinction occurred in the final year. We defined a run in which a recombinant became established in the human population as one in which its average prevalence over the final (fifth) year was $>0.0005$ (equivalent to three or four completed infections over the course of the year). In each instance, for each recombinant we calculated its frequency of appearance as the number of appearances over the number of replicates, and its frequency of establishment as the number of establishments over the number of appearances (excluding cases in which there were no appearances).

For each cross-reactivity matrix, we completed 1000 replicate runs with the parameter set at the midpoint of the parameter space defined above. To investigate the robustness of these results, for each cross-reactivity matrix we also completed 100 replicate runs with each of 81 parameter sets that define the parameter space (recombination rate $=0.0005$, 0.001 , or 0.005 ; vector half-life $=5,10$, or 15 days; duration of host infectivity $=10,20$, or 30 days; half-life of human immunity $=1000,2000$, or 3000 days). These parameter ranges are consistent with the available empirical data (McKenzie et al. 1998), and, in conjunction, lead to a wide range in overall malaria prevalence $(0.08-0.97)$ that is consistent with empirical data. In comparing frequencies of appearance, and frequencies of establishment, both within and between cross-reactivity matrices, for each parameter set we applied Fisher's exact test (Sokal and Rohlf 1981) to the contingency tables for the appropriate pairs of recombinants. To correct for multiple comparisons and a finite number of replicates (Berry and Lindgren 1990), for each such pair, across all 81 parameter sets, we compared the number of tables with calculated $P$-values $<0.05$ to the number expected under a binomial distribution with the (Bernoulli) $P$ fixed equal to the corresponding $P$-value calculated by Fisher's test. For each such pair, we ranked the average day of appearance and average prevalence for each parameter set, then compared the overall totals.

Our efforts to assemble and organize the known facts about
$P$. falciparum meiotic recombination included close examination of the literature and consultation with a dozen prominent malariologists. This process revealed an unexpected gap in current biological knowledge, in the form of irreconcilable assumptions about whether at most one, two, or four genotypes might be represented within a single $P$. falciparum oocyst. Furthermore, the adherents of each set of assumptions seemed unaware that any such disagreement exists.

All parties agree that the only diploid stage in the Plasmodium life cycle is the zygote, and that the products of meiosis are haploid. However, it appears that the electronmicroscopy studies undertaken in the 1980 s by Sinden and his colleagues ended and were considered supplanted by studies based on molecular techniques, shortly before a crucial question was resolved: "Although this study identifies the meiotic division, it is not yet established whether the fertilized zygote replicates from a diploid $(2 \mathrm{~N})$ condition to $4 \mathrm{~N}$ prior to meiosis.... We have obtained numerous pictures ... indicating that there are at least three and probably four kinetochores on each synaptonemal complex. . . . We believe that there are four chromatids in each synaptonemal complex, i.e., the zygote has indeed replicated to a $4 \mathrm{~N}$ condition prior to meiosis.... This interpretation contrasts with previous data on the related sporozoan parasite Eimeria, in which the fertilized zygote moves directly from a $2 \mathrm{~N}$ organization back to a haploid genome at the first meiotic division' (Sinden and Hartley 1985, p. 744). Most subsequent reviews of Plasmodium genetics seem to take two-step meiosis as established fact (e.g., see figure 1 in Walliker et al. 1998).

However, it is not clear that more recent results-even from single oocysts, with genes known to be single copy (e.g., MSP-1, Ranford-Cartwright et al. 1991)—exclude the possibility that Plasmodium conforms to the single-step pattern of other Sporozoa, and there may be alternative explanations for the kinetochore counts cited above. Viewed in this light, in detail, some data and language in recent studies become unexpectedly ambiguous (e.g., with respect to "levels of heterozygosity in oocysts,', Anderson et al. 2000b). Furthermore, several of our advisors maintain that the zygoteookinete-oocyst transformation leads (perhaps with rare exceptions) to exactly one genotype per oocyst. Our current, albeit tentative, view is that Sinden's hypothesis is the most likely and the one-genotype-per-oocyst hypothesis the least likely, with the phylogenetic-conformance hypothesis intermediate between these; accordingly, the model presented below is of a process in accord with Sinden and Hartley's (1985) hypothesis. However, we carried out precisely parallel studies with models that incorporate the 2 alternative hypotheses.

\section{RESULTS}

Table 1 presents results from 1000 replicate runs with each of the six matrices, from the parameter set at the midpoint of the parameter space. These results are consistent over the full range of 81 parameter sets.

First we present results for the four symmetric matrices, $\mathrm{m} 0, \mathrm{~m} 1, \mathrm{~m} 4$, and $\mathrm{m} 5$ (Fig. 2). With respect to frequencies of recombinant-form $(\mathrm{AB}, \mathrm{ab})$ appearance, the matrices rank as follows: $\mathrm{m} 0>\mathrm{m} 4>\mathrm{m} 1>\mathrm{m} 5$. With respect to frequencies of recombinant-form establishment, the matrices rank as fol- 
TABLE 1. Results on recombinants from 1000 replicate runs with each of the six matrices, with the parameter set at the midpoint of the parameter space (recombination rate 0.001 ; vector half-life 10 days; duration of host infectivity 20 days; half-life of human immunity 2000 days). Numbers of appearances and establishments refer to the number of replicates in which a recombinant appeared in a human and the number in which a recombinant persisted in the human population, respectively (see text). These results are consistent over the full range of 81 parameter sets.

\begin{tabular}{|c|c|c|c|c|c|c|c|c|}
\hline \multirow[b]{2}{*}{ Matrix } & \multirow[b]{2}{*}{$\begin{array}{c}\text { Number of } \\
\text { appearances }\end{array}$} & \multirow[b]{2}{*}{$\begin{array}{c}\text { Number of } \\
\text { establishments }\end{array}$} & \multirow[b]{2}{*}{$\begin{array}{l}\text { Average day of } \\
\text { first appearance }\end{array}$} & \multicolumn{5}{|c|}{ Average prevalence in final year } \\
\hline & & & & Overall & Initial $(\mathrm{Ab})$ & Introduced $(\mathrm{aB})$ & Mixed $(\mathrm{Ab}, \mathrm{aB})$ & $\begin{array}{l}\text { Recombinant } \\
(\mathrm{ab}, \mathrm{AB})\end{array}$ \\
\hline $\mathrm{m} 0$ & 1000 & 1000 & 883 & 0.84 & 0.37 & 0.37 & 0.14 & 0.37 \\
\hline $\mathrm{m} 4$ & 362 & 61 & 1259 & 0.61 & 0.38 & 0.38 & 0.14 & 0.00 \\
\hline $\mathrm{m} 1$ & 138 & 133 & 1394 & 0.44 & 0.28 & 0.13 & 0.00 & 0.04 \\
\hline $\mathrm{m} 5$ & 7 & 3 & 1496 & 0.40 & 0.28 & 0.13 & 0.00 & 0.00 \\
\hline $\mathrm{m} 2 . \mathrm{ab}$ & 1000 & 1000 & 815 & 0.75 & 0.37 & 0.37 & 0.14 & 0.37 \\
\hline $\mathrm{m} 2 . \mathrm{AB}$ & 313 & 50 & 1259 & - & - & - & - & 0.00 \\
\hline m3.ab & 154 & 151 & 1445 & 0.43 & 0.27 & 0.13 & 0.00 & 0.04 \\
\hline m3.AB & 20 & 12 & 1517 & - & - & - & - & 0.00 \\
\hline
\end{tabular}

lows: $\mathrm{m} 0=\mathrm{m} 1>\mathrm{m} 4=\mathrm{m} 5$. In all four symmetric matrices, the frequencies of appearance and of establishment of $\mathrm{AB}$ are indistinguishable from those of $a b$, as would be expected.

In general, matrices that lead to higher frequencies of appearance and higher frequencies of establishment are associated with earlier dates of first recombinant appearance. For instance Figure 3 illustrates the contrast between $\mathrm{m} 0$ and $\mathrm{m} 1$, again using the parameter set at the midpoint of the parameter space. The peak in the frequency in $\mathrm{m} 0$, at three months after the introduction of the new genotype $\mathrm{aB}$, occurs because no prior immunity to $\mathrm{aB}$ exists at its introduction, so the prevalence of $\mathrm{aB}$ and of mixed infections with $\mathrm{aB}$ and $\mathrm{Ab}$ rises quickly. Accordingly, the prevalence of the recombinants ab and $\mathrm{AB}$ rises soon thereafter. These peaks in prevalence produce a peak in immunity, so the frequency of $\mathrm{aB}$ and of mixed infections with $\mathrm{aB}$ and $\mathrm{Ab}$ drops quickly, as does the production of recombinants. The system then rebounds and shows an oscillating but relatively steady fall-off in the first appearances of recombinants.

With respect to the average final-year prevalence of infection overall, that is, infection with any genotype or combination of genotypes, the matrices rank as they do for frequencies of appearance: $\mathrm{m} 0>\mathrm{m} 4>\mathrm{m} 1>\mathrm{m} 5$. With respect to the final-year prevalence of the initial $(\mathrm{Ab})$ and introduced (aB) genotypes: $\mathrm{m} 0=\mathrm{m} 4>\mathrm{m} 1=\mathrm{m} 5$. With matrices $\mathrm{m} 0$ and $\mathrm{m} 4$, the prevalence of $\mathrm{Ab}$ is indistinguishable from that

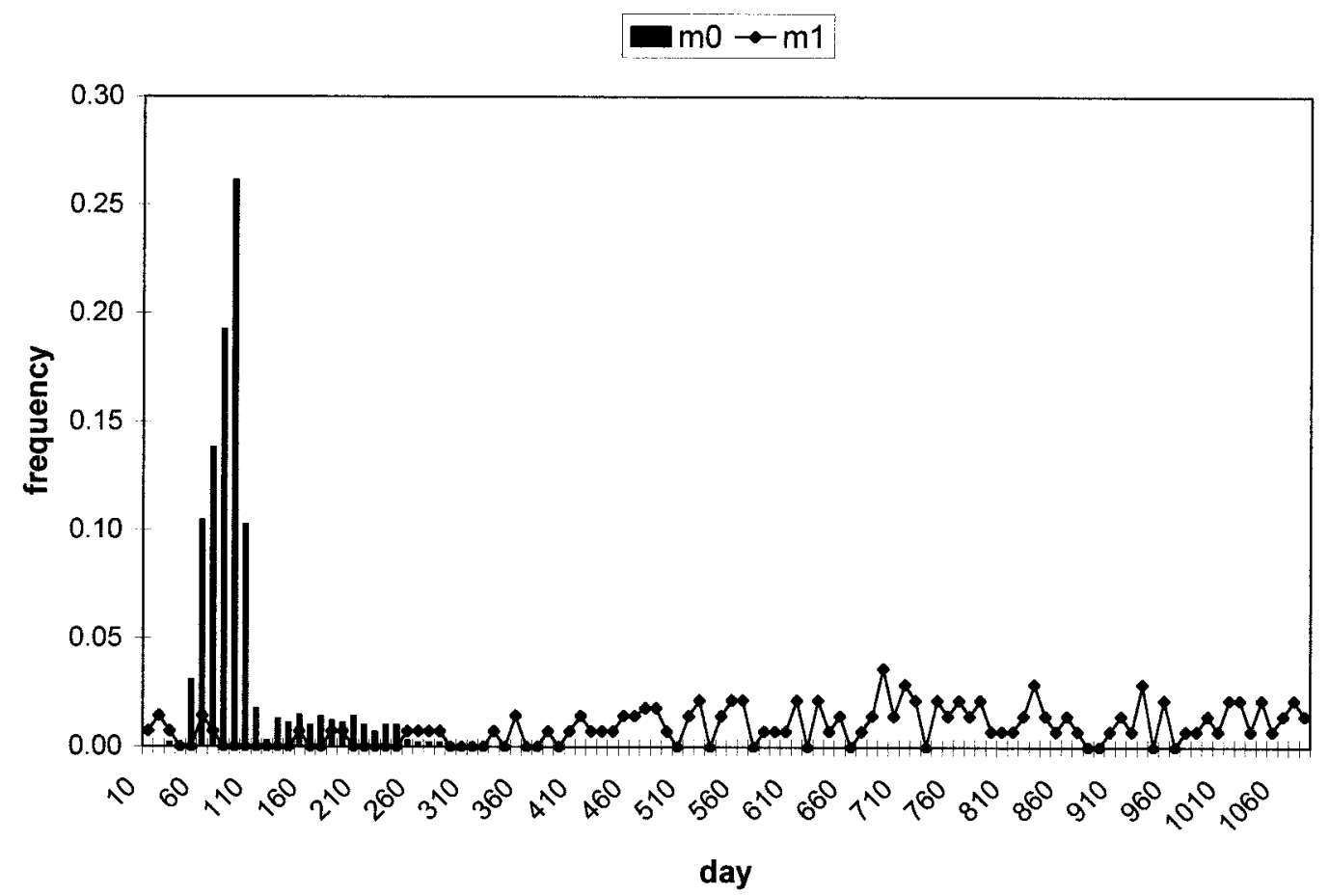

FIG. 3. The frequency distributions of the day of the first recombinant appearance in a human, over 1000 runs for matrices m0 (bars) and $\mathrm{m} 1$ (line), with the parameter set at the midpoint of the parameter space. In each run, after two years the initial genotype Ab had reached equilibrium, the genotype $\mathrm{AB}$ was introduced, and the run continues for three more years (see text). Here day 0 represents day 720 overall, that is, the day on which type $\mathrm{aB}$ was introduced. The mean first recombinant appearance for $\mathrm{m} 0$ was on day 97 and for $\mathrm{m} 1$ on day 674. 


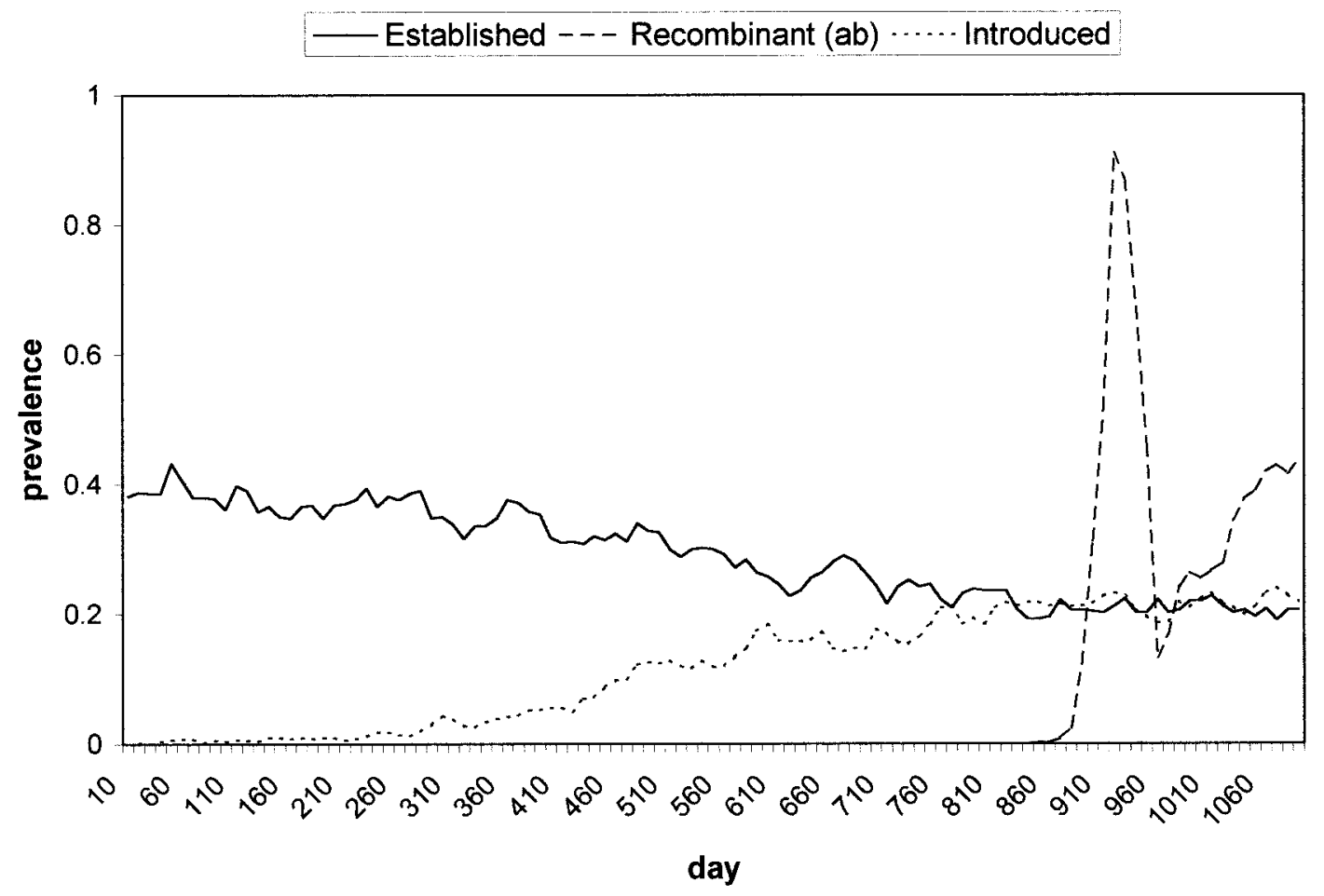

FIG. 4. The daily prevalence dynamics in a single run with matrix m3. All parameter values are as in the parameter set at the midpoint of parameter space, and day 0 represents day 720 overall, that is, the day on which type aB was introduced. The solid line represents the initial, established genotype $\mathrm{Ab}$, the dotted line the introduced $\mathrm{aB}$. Recombinant form $\mathrm{AB}$ did not appear in a human in this run; ab (dashed line) appeared in a human in $43 \%$ of runs with this parameter set, AB in only $4 \%$.

of $\mathrm{aB}$; with $\mathrm{m} 1$ and $\mathrm{m} 5$, the prevalence of $\mathrm{Ab}$ is greater than that of $\mathrm{aB}$. With matrices $\mathrm{m} 0$ and $\mathrm{m} 4$, the observed prevalence of mixed-genotype infections with $\mathrm{Ab}$ and $\mathrm{aB}$ is equal to that expected by the product of the genotype prevalences; with matrices $\mathrm{m} 1$ and $\mathrm{m} 5$, the observed prevalence is much less than that expected. With respect to the prevalence of the recombinants ( $\mathrm{AB}$ and $\mathrm{ab}$ ), the matrices rank as follows: $\mathrm{m} 0$ $>\mathrm{m} 1>\mathrm{m} 4=\mathrm{m} 5$. In all four symmetric matrices, the prevalence of $\mathrm{ab}$ is indistinguishable from that of $\mathrm{AB}$, as would be expected. The prevalence of mixed-parental-genotype infections drops dramatically with parental-parental cross-immunity; the prevalence of recombinants drops dramatically with parental-recombinant cross-immunity.

With respect to frequencies of recombinant-form appearance and establishment, dates of first recombinant appearance, and average final-year prevalences, the behavior of each of the recombinants in the asymmetric matrices $\mathrm{m} 2$ and $\mathrm{m} 3$ is indistinguishable from that of the recombinants in one of the four symmetric matrices, as follows: $\mathrm{m} 2 \mathrm{ab}=\mathrm{m} 0, \mathrm{~m} 2 . \mathrm{AB}$ $=\mathrm{m} 4, \mathrm{~m} 3 \cdot \mathrm{ab}=\mathrm{m} 1$, and $\mathrm{m} 3 \cdot \mathrm{AB}=\mathrm{m} 5$. With respect to the final-year prevalence of the initial and introduced genotypes $\mathrm{Ab}$ and $\mathrm{aB}$ respectively, $\mathrm{m} 2$ is indistinguishable from $\mathrm{m} 0$ and $\mathrm{m} 4$, and is greater that $\mathrm{m} 3$, which is indistinguishable from $\mathrm{m} 1$ and $\mathrm{m} 5$. With $\mathrm{m} 2$, the prevalence of $\mathrm{Ab}$ is indistinguishable from that of $\mathrm{aB}$; with $\mathrm{m} 3$, the prevalence of $\mathrm{Ab}$ is greater than that of $\mathrm{aB}$. With matrix $\mathrm{m} 2$, the observed prevalence of mixed-genotype infections with $\mathrm{Ab}$ and $\mathrm{aB}$ is equal to that expected by the product of the genotype prevalences; with matrix $\mathrm{m} 3$, the observed prevalence is much less than that expected. In accord with equivalencies noted above, in both asymmetric matrices the frequency of appearance, frequency of establishment, and final-year prevalence of ab are greater than those of $\mathrm{AB}$, and the overall malaria prevalence is intermediate between the corresponding symmetric matrices.

Figure 4 illustrates the dynamics in a single run with matrix $\mathrm{m} 3$, in which all genotypes but the recombinant ab are crossreactive. Typically, the recombinant form $\mathrm{AB}$ failed to appear in a host during this run. Following its introduction, the prevalence of genotype aB increases slowly, because the host population has a high level of immunity to genotype Ab. The rising prevalence of $\mathrm{aB}$ suppresses the prevalence of $\mathrm{Ab}$. The immunologically independent recombinant $a b$ appears for the first time 28 months after the introduction of $\mathrm{aB}$ and displays dynamics typical of a genotype in a naive host population. Its sudden rise in prevalence reflects the nearly simultaneous infection of a large fraction of the host population, which synchronizes host immunity and produces a rapid decline in prevalence to a level well below its eventual equilibrium.

In sum, cross-reactivity between the parental types produces lower frequencies of appearance. Cross-reactivity between the parental and recombinant forms also produces lower frequencies of appearance, although with less marked effect, and, unlike cross-reactivity between the parental types, it produces dramatically lower frequencies of establishment. With cross-reactivity both between the parental and recombinant forms and between the parental types, frequencies of establishment are greater than with only parental-recombinant cross-reactivity. Parental-recombinant cross-reactivity influences overall prevalence solely through its influence on the prevalence of recombinants. Parental-parental cross-re- 
TABLE 2. Results on recombinants from 1000 replicate runs with each of the six matrices (parameter set as in Table 1), with models that incorporate the two alternative hypotheses about the maximum number of parasite genotypes in a single oocyst. The appearance and establishment figures are given as ratios in relation to the corresponding figures in Table 1. We do not show the average day of first appearance, which for the one-genotype model ranged from three months earlier to three months later, and for the two-genotype model from four months earlier to one month later than the four-genotype model. All average final-year prevalence figures were within 0.01 of those in Table 1 . Results for each model are consistent across all 81 parameter sets.

\begin{tabular}{lccccc}
\hline \hline & \multicolumn{2}{c}{ One genotype/oocyst } & & \multicolumn{2}{c}{ Two genotypes/oocyst } \\
\cline { 2 - 3 } \cline { 5 - 6 } Matrix & $\begin{array}{c}\text { Number of } \\
\text { appearances }\end{array}$ & $\begin{array}{c}\text { Number of } \\
\text { establishments }\end{array}$ & & $\begin{array}{c}\text { Number of } \\
\text { appearances }\end{array}$ & $\begin{array}{c}\text { Number of } \\
\text { establishments }\end{array}$ \\
\hline $\mathrm{m} 0$ & 1.00 & 1.00 & & 1.00 & 1.00 \\
$\mathrm{~m} 4$ & 0.46 & 0.39 & & 0.92 & 0.89 \\
$\mathrm{~m} 1$ & 0.72 & 0.80 & & 1.07 & 1.10 \\
m5 & 0.86 & 1.00 & & 1.71 & 1.67 \\
m2.ab & 1.00 & 1.00 & & 1.00 & 1.00 \\
m2.AB & 0.51 & 0.48 & & 1.03 & 110 \\
m3.ab & 0.60 & 0.61 & & 1.05 & 1.04 \\
m3.AB & 0.35 & 0.33 & & 0.80 & 0.67 \\
\hline
\end{tabular}

activity influences all components of overall prevalence, but the frequency of establishment and prevalence of recombinants are higher with parental-parental than with parentalrecombinant cross-reactivity.

Table 2 presents results from two alternative models, incorporating the hypotheses that there is at most one genotype or at most two genotypes in a single $P$. falciparum oocyst. Although differences from the four-genotype-per-oocyst model are quantitative rather than qualitative, they are sufficiently striking to merit attention.

\section{Discussion}

Our results argue that rates of parasite evolution are influenced by cross-reactivity between parental genotypes and between recombinant and parental genotypes; each form of cross-reactivity exerts strong but somewhat different effects. Outcrossed recombinants arise in mosquitoes primarily from mixed parental-genotype infections in humans, the frequencies of which are influenced most strongly by cross-reactivity between the parental genotypes. Frequencies of recombinant appearance also decline with the presence of parental-recombinant cross-reactivity because recombinants generated in the mosquito are likely to encounter an effective immune response when inoculated into a host. Once a novel recombinant emerges in a human population, its probability of persistence is influenced most strongly by cross-reactivity with its parental forms. In this context, although cross-reactivity between the parental genotypes reduces the prevalence of each, and thus the frequency of outcrossing, it also reduces the probability that a novel recombinant will be inoculated into an effectively immune host.

Novel recombinants are most likely to derive from immunologically novel invaders, not because the invading parental genotype produces a novel protein per se, but because it bypasses the bottlenecks in transmission that govern the dynamics of parasite recombination. It is important to note that the structure of cross-immunity between the model genotypes here is in no way necessarily tied to or dependent on the loci or alleles involved in recombination. At least two of the cross-reactivity matrices could be considered to represent relationships among alleles of antigen-producing loci, however: allele- and locus-specific cross-reactivity involving both alleles at both loci (m4) or allele-specific cross-immunity involving one allele at each locus (m2). Matrix m3 might represent a relationship in which the antigenic products of one allele at each locus are cross-reactive. Nonetheless, our results suggest that the effects of cross-reactivity on $P$. falciparum population genetics extend beyond specific antigen-producing loci; for example, a drug-resistant recombinant might be less likely to emerge were its parental genotypes cross-reactive.

Values for some parameters in our model are not known as yet, and the values of others are known to vary widely in nature. Accordingly, we examined ranges of values that correspond to the available empirical data. Our comparisons of cross-reactivity matrices produced results robust over all of the parameter sets and indicated that in this context crossreactivity structures influence the emergence, persistence, and prevalence of novel genotypes more than do parameter sets. However, it is also clear that important changes in system dynamics can proceed from specific changes at the level of the parameter values themselves. For instance, in accord with Tanaka and Feldman (1999), we note that changes in recombination rate affect the success of an invading genotype primarily by affecting the magnitude of the advantage or disadvantage conferred by its position in a particular crossreactivity (interference) structure. In broad terms, our results support the conclusions of several other recent modelers, in particular that immune-mediated relationships among genotypes may shape and be shaped by $P$. falciparum evolution. Thus, it seems possible that intervention could disrupt these ecological relationships so as to release vaccine-evading variants from competitive suppression (Gupta et al. 1997) or facilitate the spread of drug resistant mutants (Hastings and Mackinnon 1998).

Our model has a number of shortcomings, some of which might be addressed by incorporating more detailed models of within-host parasite dynamics (McKenzie and Bossert 1997b, 1998). The model considers only binary densities in the transmission of genotypes, either as gametocytes or sporozoites, and, in more general terms, although our operational definition of immune functions is adequate for the present purpose, we look forward to much more detailed, mechanistic representations of immune responses.

More detailed immune-response representations will be essential to models that connect clinical disease to malaria infection. For instance, in sub-Saharan Africa, malaria mortality and morbidity are generally low among those adults and older children exposed to at least five infections per year; high mortality is limited to infants and young children. Malaria prevalence is typically high in both groups, and the clinical difference is said to arise from cumulative exposure to the antigenic repertoire of the parasite, leading to greater cross-reactive immunity with age. However, an alternative hypothesis attributes the difference to intrinsic age-related immune factors independent of cumulative exposure, shifting the focus of risk much more toward the host than the parasite (Baird 1995). It is critical that this question be resolved, in 
part because most vaccines under development to reduce mortality in Africa expect to mimic the naturally acquired immunity of adults.

We also expect that the unanticipated questions about meiosis in P. falciparum-concerning the mechanics of parasite genotype packaging in oocysts, and thus the biological units subject to survivorship and selection (Vaughan et al. 1992)will be resolved. In the present context, differences between the three hypotheses have quantitative rather than qualitative consequences; although none affect our conclusions here, their implications for other avenues of malaria research are significant.

The slow and imperfect acquisition of immunity in human malaria is usually attributed to the antigenic diversity of the parasite, and the antigenic diversity of the parasite is usually attributed to its obligate sexual recombination in Anopheles. Our current knowledge of $P$. falciparum genetics is heavily biased toward a few drug-resistance or strongly immunogenic vaccine-candidate genes, however, and even in these we know little about linkage and nothing about mutation rates. Furthermore, it may be that evolutionary trajectories differ among antigen-producing loci (Escalante et al. 1998) and even among allelic families at a single antigen-producing locus (Felger et al. 1997). Longitudinal studies of infected individuals have begun to reveal complex day-to-day dynamics involving variously defined $P$. falciparum allelic types (Daubersies et al. 1996; Farnert et al. 1997), but we do not understand how parasite dynamics within individuals relate to those in human and vector populations (Arnot 1998; Druilhe et al. 1998).

Malaria intervention programs should be rationally designed, executed, and evaluated, and so should encompass a detailed understanding of the population-genetic and crossreactivity structures of $P$. falciparum populations, how these structures interact in the evolution of the parasite, and how such interactions may vary with place, time, and the intervention programs themselves. Biased frequencies of parasite genotypes transmitted to mosquitoes under drug pressure will be crucial to the spread of resistance, and, under vaccine pressure, to the spread of evasive antigenic variants. It would be extremely premature to expect a deep understanding of any system of this complexity on the basis of the meager current data. Furthermore, a deep understanding will require not only a great deal more data, but an array of formal explanatory models incorporating as many of relevant factors as possible, in a realistic but comprehensible manner. Our discrete-event implementation cannot replace more abstract approaches or preempt more precise site-specific simulations, but can serve as a bridge between them, thus allowing integration and comparison of data from different places, times, and disciplines.

\section{ACKNOWLEDGMENTS}

We gratefully acknowledge the contributions of C. Gaab, K. Hoffman, R. L. Merriam, J. Moore, E. Pickens, M. Riley, J. Simek, B. C. Sorkin, N. Vecchione, and two anonymous reviewers. FEM is supported by a NIH National Research Service Award, MUF by FAPESP and the UNDP/World-
Bank/WHO Special Program for Research and Training in Tropical Diseases.

\section{Literature Cited}

Anderson, T. J. C., B. Haubold, J. T. Williams, J. G. Estrada-Franco, L. Richardson, R. Mollinedo, M. Bockarie, J. Mokili, S. Mharakurwa, N. French, J. Whitworth, I. D. Velez, A. Brockman, F. Nosten, M. U. Ferreira, and K. P. Day. 2000a. Microsatellites reveal a spectrum of population structures in the malaria parasite Plasmodium falciparum. Mol. Biol. Evol. 17:1467-1482.

Anderson, T. J. C., R. E. L. Paul, C. A. Donnelly, and K. P. Day 2000b. Do malaria parasites mate non-randomly in the mosquito midgut? Genet. Res. 75:285-296.

Arnot, D. 1998. Clone multiplicity of Plasmodium falciparum infections in individuals exposed to variable levels of disease transmission. Trans. R. Soc. Trop. Med. Hyg. 92:580-585.

Ayala, F. J., A. A. Escalante, A. A. Lal, and S. M. Rich. 1998. Evolutionary relationships of human malaria parasites. Pp. 285300 in I. W. Sherman, ed. Malaria. ASM Press, Washington, D.C.

Baird, J. K. 1995. Host age as a determinant of naturally acquired immunity to Plasmodium falciparum. Parasitol. Today 11: $105-111$.

12000. Resurgent malaria at the milennium. Drugs 59: 719-743.

Berry, D. A., and B. W. Lindgren. 1990. Statistics. Brooks-Cole, Pacific Grove, CA.

Buckling, A., L. C. Ranford-Cartwright, A. Miles, and A. F. Read. 1999. Chloroquine increases Plasmodium falciparum gametocytogenesis in vitro. Parasitology 118:339-346.

Carter, R., and P. M. Graves. 1988. Gametocytes. Pp. 253-306 in W. H. Wernsdorfer and I. McGregor, eds. Malaria. Churchill Livingstone, Edinburgh.

Daubersies, P., S. Sallenave-Sales, S. Magne, J.-F. Trape, H. Contamin, T. Fandeur, C. Rogier, O. Mercereau-Puijalon, and P. Druilhe. 1996. Rapid turnover of Plasmodium falciparum populations in asymptomatic individuals living in a high transmission area. Am. J. Trop. Med. Hyg. 54:18-26.

Druilhe, P., P. Daubersies, J. Patarapotikul, C. Gentil, L. Chene, T. Chongsuphajaisiddhi, S. Mellouk, and G. Langsley. 1998. A primary malarial infection is composed of a very wide range of genetically diverse but related parasites. J. Clin. Invest. 101: 2008-2016.

Escalante, A. A., A. A. Lal, and F. J. Ayala. 1998. Genetic polymorphism and natural selection in the malaria parasite Plasmodium falciparum. Genetics 149:189-202.

Farnert, A., G. Snounou, I. Rooth, and A. Bjorkman. 1997. Daily dynamics of Plasmodium falciparum subpopulations in asymptomatic children in a holoendemic area. Am. J. Trop. Med. Hyg. 56:538-547.

Felger, I., V. M. Marshall, J. C. Reeder, J. A. Hunt, C. S. Mgone, and H. P. Beck. 1997. Sequence diversity and molecular diversity of the merozoite surface antigen 2 of Plasmodium falciparum. J. Mol. Evol. 45:154-160.

Ferreira, M. U., Q. Liu, M. Kimura, B. T. Ndawi, K. Tanabe, and F. Kawamoto. 1998. Allelic diversity in the merozoite surface protein-1 and epidemiology of multiple-clone Plasmodium falciparum infections in Northern Tanzania. J. Parasitol. 84: 1286-1289.

Gilbert, S. C., M. Plebanski, S. Gupta, J. Morris, M. Cox, M. Aidoo, D. Kwiatkowski, B. M. Greenwood, H. C. Whittle, and A. V. S. Hill. 1998. Association of malaria parasite population structure, HLA, and immunological antagonism. Science 279: 1173-1177.

Gupta, S., N. M. Ferguson, and R. M. Anderson. 1997. Vaccination and the population structure of antigenically diverse pathogens that exchange genetic material. Proc. R. Soc. Lond. B 264: 1435-1443.

Haight, F. A. 1967. Handbook of the Poisson distribution. John Wiley, New York.

Haji, H., T. Smith, J. D. Charlwood, and J. H. Meuwissen. 1996. 
Absence of relationships between selected human factors and natural infectivity of Plasmodium falciparum to mosquitoes in an area of high transmission. Parasitology 113:425-431.

Hastings, I. M., and M. J. Mackinnon 1998. The emergence of drugresistant malaria. Parasitology 117:411-417.

Healer, J., D. McGuinness, R. Carter, and E. Riley. 1999. Transmission-blocking immunity to Plasmodium falciparum in malaria-immune individuals is associated with antibodies to the gamete surface protein Pfs230. Parasitology 119:425-433.

Macdonald, G. 1957. The epidemiology and control of malaria. Oxford Univ. Press, London.

McKenzie, F. E., and W. H. Bossert. 1997a. Mixed-species Plasmodium infections of Anopheles (Diptera: Culicidae). J. Med. Entomol. 34:417-425.

1997b. The dynamics of Plasmodium falciparum bloodstage infection. J. Theor. Biol. 188:127-140.

. 1998. The optimal production of gametocytes by Plasmodium falciparum. J. Theor. Biol. 193:419-428.

1999. Multispecies Plasmodium infections of humans. J. Parasitol. 85:12-18.

McKenzie, F. E., R. C. Wong, and W. H. Bossert. 1998. Discreteevent simulation models of Plasmodium falciparum malaria. Simulation 71:250-261.

1999. Discrete-event simulation models of mixed-phenotype Plasmodium falciparum malaria. Simulation 73:213-217.

Paul, R. E. L., A. Brockman, R. N. Price, C. Luxemburger, N. J. White, S. Looareesuwan, F. Nosten, and K. P. Day. 1999. Genetic analysis of Plasmodium falciparum infections on the northwestern border of Thailand. Trans. R. Soc. Trop. Med. Hyg. 93: 587-593.

Pringle, G. 1966. A quantitative study of naturally-acquired malaria infections in Anopheles gambiae and Anopheles funestus in a highly malarious area of East Africa. Trans. R. Soc. Trop. Med. Hyg. 60:626-632.

Ranford-Cartwright, L. C., P. Balfe, R. Carter, and D. Walliker. 1991. Genetic hybrids of Plasmodium falciparum identified by amplification of genomic DNA from single oocysts. Mol. Biochem. Parasitol 49:239-244.

Sinden, R. E., and R. H. Hartley. 1985. Identification of the meiotic division of malarial parasites. J. Protozool. 32:742-744.

Snounou, G., X. Zhu, N. Siripoon, W. Jarra, S. Thaithong, K. N. Brown, and S. Viriyakosol. 1999. Biased distribution of $m s p l$ and msp2 allelic variants in Plasmodium falciparum populations in Thailand. Trans. R. Soc. Trop. Med. Hyg. 93:369-374.

Sokal, R. R., and F. J. Rohlf. 1981. Biometry. W. H. Freeman, New York.

Tanaka, M. M., and M. W. Feldman. 1999. Theoretical considerations of cross-immunity, recombination and the evolution of new parasitic strains. J. Theor. Biol. 198:145-163.

Toure, Y. T., O. Doumbo, A. Toure, M. Bagayoko, M. Diallo, A. Dolo, K. D. Vernick, D. B. Keister, O. Muratova, and D. C. Kaslow. 1998. Gametocyte infectivity by direct mosquito feeds in an area of seasonal malaria transmission: implications for Bancoumana, Mali, as a transmission-blocking vaccine site. Am. J. Trop. Med. Hyg. 59:481-486.

Vaughan, J. A., B. H. Noden, and J. C. Beier. 1992. Population dynamics of Plasmodium falciparum sporogony in laboratoryinfected Anopheles gambiae. J. Parasitol. 78:716-724.

Walliker, D., H. Babiker, and L. Ranford-Cartwright. 1998. The genetic structure of malaria parasite populations. Pp. 235-252 in I. W. Sherman, ed. Malaria. ASM Press, Washington, D.C.

Corresponding Editor: M. Riley 